Vietnam Journal of Mechanics, VAST, Vol.31, No. 2 (2009), pp. 97-102

\title{
DUALITY IN PROCESS OF NONCOMMUTATIVE DEFORMATION AND TOPOLOGICAL NATURE OF CHEREPANOV-RICE INTEGRAL
}

\author{
Trinh Van Khoa \\ Hanoi Architectural University
}

\begin{abstract}
In this paper it is showed, that for the noncommutative deformation simultaneously there exist also loading deformation $H$, and unloading deformation $H^{v}$. The real deformation is a combination of these types of deformations. The criterion of destruction $J$ reflects topological character of medium, i.e. it defines properties of symmetry of medium at destruction. It is possible to tell, that during destruction the energy is released not continuously but and discretely. This situation is reflected through topological number $Q$ or number of unloading, connected to him.
\end{abstract}

\section{INTRODUCTION}

As known, after removal of loading an elastic body always comes back in an initial state. The given definition of elasticity is a little simplified. If, for example, the stress exceeds a limit of elasticity, the dependence between loading and deformation ceases to be linear and depends on the order of the application of loading [1]. For simplicity, it is supposed, that the equation of a curve

$$
\sigma=\Phi(e)
$$

is obtained at any program of the application of load, when the stress monotonously grows. Nevertheless for real materials it is more complicated. Let we have finished in loading up to a point $A=\left(\sigma^{\prime}, e^{\prime}\right)$ of area of plasticity. After the unloading is made, the stress $\sigma$ decreases to zero. In this process it is reflected not only plastic behavior of material, but also elastic. More careful experiments have shown, that the law of unloading is not described precisely by straight line. Replacing it by its closest straight line, we find, that its inclination does not correspond in accuracy to the initial module of elasticity. For polymeric materials, and also at composite materials, for example, fibreglasses, the law of unloading differs from the Hooke's law very essentially. Apparently, the defects are formed in material during loading. Naturally there is a question: Whether exists duality of loading and unloading states? And in general, whether exists duality during plastic deformation? Let's consider this problem in given paper. The paper is organized as follows. The section 2 contains shelf-organization characters of the process of plastic deformation. In section 3 we present noncommutative model of plastic deformation. The section 4 contains the relations between the loading and unloading. In the section 5 we present some remarks. 


\section{THE PROCESS OF PLASTIC DEFORMATION AS A PROCESS OF SHELF-ORGANIZATION}

As known, under action of loading the material passes in plastic state. All data of experiments show, that in this state the stress and the strain are in strongly fluctuation. And what's more, the stress - strain diagram $\sigma=f(e)$ is similar with the pressure - volume diagram $P=f(V)$ in a liquid [2]. On the other hand, the local phase transition in the end of a crack is observed [3-5]. Thus, it is possible to consider the state of destruction as a state of transition, in which a new structure arises spontaneously [6]. The density of the distribution of probability of transition to plasticity is a solution of the Fokker - Planck equation. However, instead of the Fokker - Planck equation we can use the Schrodinger equation when using the potential character of deformation process. It means, that it is possible to consider the process of plastic deformation as the process with supersymmetry. In this situation the strain tensor is considered in the role of the parameter of order. In work [1] A.A. Iliushin has presented on discussion the concept of a trajectory of deformation in space $E_{5}$. Actually, the space $E_{5}$ is a layer in the bundle of deformation [6]. It means, that we can consider the field of deformation as a gauge field, in which generalized Iliushin trajectory of deformation is a section of a bundle of deformation. When we identify the generalized Iliushin trajectory of deformation with a knot we have shown topological character of the deformation process connected with Chern - Simonce - Witten invariant

. Thus, the density of distributions of probability of transition to plasticity is received in the form $[6]$ :

$$
Z=\int \exp \left[-k S_{c s}(e)\right] \mathcal{D} e
$$

here $k$ is the factor of the elasticity, and $S_{C S}$ is Chern - Simons - Witten action.

$$
S_{c s}=\int \Gamma_{c s}
$$

where $\Gamma_{c s}$ is the Chern - Simons form for variety of deformation.

\section{THE NONCOMMUTATIVE MODEL OF PLASTIC DEFORMATION}

Thank to the spontaneous breakdown of symmetry we can receive laminar structure in process of thermomechanical deformation [6]. And from this mechanism we can describe two processes of loading and unloading with their topological character, which will be considered as a dual processes. The process of deformation in the end of a dislocation line and the cracks are examples of unloading, which we would like to consider here. These processes of unloading and at the same time liberating of energy of deformation were used in model of seismology. Nevertheless it is necessary to notice, that the nature of the process of plastic deformation is noncommutative. So, we suppose that a following process of deformation is given $P=\left\{E_{i j}(x, t)\right\}$. It is noncommutative space of deformation. The deformation wave is a noncommutative wave. Besides, it is possible to consider a scalar field describing the action of a defect on deformation process[7]. For the complete description of the distributions of a deformation wave it is necessary for us to use the space being tensor product $C\left(R_{4}\right) \otimes M_{n}$, where $C\left(R_{4}\right)$ is an algebra of smooth functions determined on usual space - time and $M_{n}$ is an algebra of $n \times n$ matrixes. Now our task is to construct 
noncommutative bundles of deformation over $C\left(R_{4}\right) \otimes M_{n}$. This model of deformation was constructed in [6]. Here we admit the following connection $\omega=A_{\mu} d x^{\mu}+\left(B_{k}-i E_{k} \otimes 1\right) \theta^{k}$, $A_{\mu}$ is function in $V$, receiving value in $M_{n}(C) \times M_{r}(C), \mu \in\{0,1 . ., s\}, B_{k}$ is function over $V$, having the value in $M_{n}(C) \times M_{r}(C)$. Thus, the action will be expressed in the form:

$$
\begin{aligned}
S= & -\int_{R^{s+1}} \frac{1}{4 n} \operatorname{Tr}\left(F_{\mu \nu} F^{\mu \nu}\right)+\frac{1}{2 n} \operatorname{Tr}\left[\left(\nabla_{\lambda} \phi_{k}\right)\left(\nabla^{\lambda} \phi^{k}\right)\right] \\
& +\frac{1}{4 n} \sum \operatorname{Tr}\left[\left[\phi_{k}, \phi_{l}\right]-\mathbf{m} \sum C_{k l m} \phi_{m}\right]^{2}
\end{aligned}
$$

In this case $F_{\mu \nu}^{a}=\partial_{\mu} A_{\nu}-\partial_{\nu} A_{\mu}+\left[A_{\mu}, A_{\nu}\right], \phi_{k}=\mathbf{m} B_{k}, \nabla_{\lambda} \phi_{k}=\partial_{\lambda} \phi_{k}+\left[A_{\lambda}, \phi_{k}\right]$

"Magnetic monopol" arisen during evolution of gauge fields, actually is the deformation of the unloading. Really, from the action $S$ we shall receive the equation of motion.

$$
\delta S=0 .
$$

This is a nonlinear equation, the solution of which with limited energy is important for research of the topological character of system. However, first of all it is possible to have some informations on its properties. For this purpose it is necessary to find the expression of energy of system from Lagrangian with the condition $A_{0}^{a}=0$. It looks like as

$$
\begin{aligned}
W= & -\int_{R^{s}} d^{s} x \frac{1}{4 n} \operatorname{Tr}\left(F_{\mu \nu} F^{\mu \nu}\right)+\frac{1}{2 n} \operatorname{Tr}\left[\left(\nabla_{\lambda} \phi_{k}\right)\left(\nabla^{\lambda} \phi^{k}\right)\right] \\
& +\frac{1}{4 n} \sum \operatorname{Tr}\left[\left[\phi_{k}, \phi_{l}\right]-\mathbf{m} \sum C_{k l m} \phi_{m}\right]^{2}
\end{aligned}
$$

It is the form of Cherepanov - Rice integral without kinetic energy. This energy achieves the minimum ( and equal to zero ) at

$$
A_{0}(x)=0, \phi_{i}(x)=\frac{1}{2} \mathbf{m} E_{i}, \nabla_{\lambda} \phi^{i}=0
$$

In special case the last equation returns turns to the equation $\partial_{i} \phi^{a}=0$. There are two gauge orbits. Suppose that $\Omega_{0}$ is vacuum according to an orbit $\left(A_{\mu}=0, \phi_{k}=0\right)$, and $\Omega_{1}$ is vacuum according to the orbit $\left(A_{\mu}=0, \phi_{k}=i \mathrm{~m} E_{k}\right)$. Thus, the above condition is carried out for each orbit. In the case, when required the finiteness of deformation energy $W<\infty$, then we have following result. Because the condition of finiteness $W<\infty$ includes the fact that the deformation field goes quickly to the some configuration of field in which $W=0$ on spatial infinity, since

$$
r^{3 / 2} \nabla_{i} \phi \rightarrow 0, \phi \phi \rightarrow F \text { when } r \equiv|x| \rightarrow \infty
$$

For getting the character of the field $A$ we shall present $\nabla_{i}$ in spherical coordinates $\{r, \theta, \phi\}, \theta$ is the component determined by expression $\left(\nabla \phi^{a}\right)_{\theta}=\frac{1}{r} \frac{\partial \phi^{a}}{\partial \theta}+g C^{a b c} A_{\theta}^{b} \phi^{c}$. Then for giving above condition the field $A_{\theta}^{b}$ cannot satisfy condition $A_{\theta}^{b}=\frac{1}{r} A(\mathbf{n})$, where $A(\mathbf{n})$ depends only on a spatial corner: $\sum n_{i}^{2}=1$. Other components have similar character. Thus, there are available boundary conditions in the infinity

$$
A_{\mu}^{a}=\frac{1}{r} A(\mathbf{n}), \phi^{a}=\frac{1}{2} \mathbf{m} E_{a}, r^{3 / 2} \nabla_{i} \phi=0 .
$$


So, we shall receive following topological index

$$
Q=\int d^{3} x K_{0}
$$

and topological current looks like

$$
K_{\mu}=\frac{1}{8 \pi} \operatorname{Tr}\left(\partial^{\mu} \hat{\phi}^{a} \partial^{\varrho} \hat{\phi}^{b}\right) \partial^{\sigma} \hat{\phi}^{c},
$$

where $\hat{\phi}_{a}=\phi^{a} /|\phi|,|\phi|=[\operatorname{Tr}(\phi \phi)]^{1 / 2}$. The "charge" of unloading looks like $m=$ $\int \frac{K_{0}}{g} d^{3} x=Q / g$. In the case $n, r=1$ we shall receive usual t'Hoof- Polyakov monopol.

\section{THE RELATION OF LOADING AND UNLOADING}

Let consider the duality between the loading and the unloading. Suppose that the deformation medium has the symmetry $G$. For simplicity let's assume, that $G$ is semisimple group. Then the spontaneous breakdown of symmetry $G \rightarrow H \subset G$ will appear in the deformation process. As a result it is necessary to determine the group $H^{v}$ of the process of unloading. For this purpose we shall investigate the structure of the component $A(\theta)$. In commutative case in [8] one approved, that a component $A(\theta)$ of monopole is fully determined through the Higgs field $\phi$. In our noncommutative case on the basis of the equation of motion we also receive the same statement. Furthermore, from the requirement of finiteness of deformation energy as in the classical deformation theory we receive the topological number [6]. On the other hand in the classical fracture theory there is the Cherepanov - Rice integral which is one of the invariants. We shall copy this result in the equivalent form, which does not diminish the generality of the problem:

$$
\exp (4 \pi i \in A(\theta))=1 \text {, }
$$

where $\epsilon$ is a constant of the connection of defect with medium. Let's $\mathcal{L}(H)$ is Lie algebra of the group $H$, and $T_{1}, T_{2} \ldots T_{r}$ are its generators. Thus, according to the theory of Lie group and Lie algebra [10] it is possible to find gauge maps $S \in H$, so that

$$
\epsilon A(\theta)=S \sum_{i=1}^{r} \beta_{i} T_{i} S^{-1} .
$$

The system $\beta_{1}, \beta_{2} \ldots \beta_{r}$ is named as the weight of unloading state. Using this concept, it is possible to rewrite above condition of "quantization" as

$$
\exp \left(4 \pi i \sum_{i=1}^{r} \beta_{i} T_{i}\right)=1 .
$$

If $H$ is the group for loading, then it is necessary to search the group $H^{v}$ for unloading. Thus, our purpose is to deform a system of the weight of group $H^{v}$, satisfying above mentioned condition, for this we use method in [9]. Suppose that, for group $H$ there is a system of roots $\alpha \in \Phi(H)$, then the system of roots $\omega$ of universal enveloping group $\tilde{H}$ will look like

$$
\Lambda(\tilde{H})=\left\{\omega ; 2 \omega \cdot \frac{\alpha}{\alpha^{2}} \in Z ; \alpha \in \Phi(H),\right.
$$


where $\Phi(H)$ is the system of roots of group $H$. First of all we deform the system $\beta_{i}$ such that the condition was satisfied:

$$
\widetilde{\exp }(4 \pi i \beta . T) \in Z(\tilde{H}),
$$

where $Z(\tilde{H})$ is the center. From that point we have a system of dual roots $\Phi^{v}(H)=$ $\left\{\alpha^{v}=N^{-1} \frac{\alpha}{\alpha^{2}} ; \alpha \in \Phi(H)\right\}$. For the elementary case, when $H$ is a simple group, $N$ will be a number $N^{2}=\left(\sum \frac{1}{\alpha^{2}}\right) / \sum \alpha^{2}$. Since, the system of the weight $\Lambda\left(\tilde{H}^{v}\right)$ has the form $: \Lambda\left(\tilde{H}^{v}\right)=\{\omega ; 2 \omega N \alpha \in Z ; \alpha \in \Phi(H)\}$, according to Lee algebra, the greatest system of the weight $\beta$, satisfying the condition $2 \alpha . \beta \in Z, \alpha \in \Phi(H)$, is written in the form $\beta=N \omega, \omega \in \Lambda\left(\tilde{H}^{v}\right)$. The last step in our task is to look for the system $\beta$ carrying out the condition $\exp \left(4 \pi i \sum \beta_{i} T_{i}\right)=1$ or the condition $2 \beta . \omega \in Z, \omega \in \Lambda(H)$. The general form of the system $\beta$, carrying out the described above condition, looks like $N^{-1} \beta \in \Lambda\left(H^{v}\right)$. This system determines all characters of the group $H^{v}$.

\section{DISCUSSION}

According to our noncommutative model of deformation the process of destruction of medium is following. Suppose that a crack (defect) is existed in medium with the group of symmetry $G$. Under the action of loading the medium with cracks passes in the state of destruction (the state of phase transition). Thus there is a spontaneous breakdown symme$\operatorname{try} G \rightarrow H$. More precisely, it is the process $G \rightarrow H \otimes H^{v}$. It means, that for the loading $H$, there unloading $H^{v}$ exist simultaneously. The real deformation is a combination of these types of deformations. The presence of topological objects creates a difference between loading and unloading. From the requirement of finiteness of the deformation energy and under influence of spontaneous breakdown of symmetry we shall receive the fact, that the space of deformation is the bundle and the integral of deformation energy is an integral of Cherepanov - Rice type. Simultaneously it notes that the function under integral is the potential of deformation. In the classical mechanics of destruction Cherepanov - Rice integral is considered as the criterion of destruction. Actually, on our noncommutative model the criterion of destruction reflects topological character of medium, i.e. defines properties of symmetry of medium at destruction. It is possible to tell, that during destruction the energy released is not continuous but discrete. This situation is reflected through topological number $Q$ or number of unloading, connected to it.

\section{ACKNOWLEDGEMENTS}

This paper is completed with the financial support from the National Council of Natural Sciences.

\section{REFERENCES}

[1] A. A. Iliushin, Mechanics of Continuous media, MGU 1994;

[2] V. D. Klusnikov, Physical and Mathematical Bases of Durability and Plasticity, MGY 1994;

[3] L.I. Sedov, Mechanics of Continuous media, M. Science, 1973; 
[4] M. Ausloos, Phase transitions theory approach to fracture of materials, Solid Stat. Comm. 59 (1986) (6) 401-404.

[5] V. G. Gargin, Thermal destruction of synthetic almaz, Superfirm materials (1982) (2) 17-20.

[6] V. A. Pesin, N. N. Tkachenko, L. I. Feldchuk, Kinetic of polimorf prevasenie viursitopodobnogo nitrida bora pri vusokich davleniach i temperaturach (in Russian), JFCh. 53 (2) (1979) 2794.

[7] Li-Shing Li, R. J. Pabst, On the phase transition in medium with fracture, Material Sci. 15 (1980) (10) 2861.

[8] V. K. Trinh, A model of a crack in the vicinity o phase transition, Report of RAN 372 (2000) (4) 473.

[9] V. K. Trinh, Noncommutative plastic deformation and the essence of the Ilyushin's isotropy postulate, The report of RAN 378 (2001) (3) 343;

[10] V. K. Trinh, Analysis and its The application, Thesis of the reports of VZMS-2000 conference; mathematical modeling in natural and humanitarian sciences, Voronezh, January 20-27, 2000; cond-mat/9907290; Noncommutative thermomechanical deformation, Bulletin MGU: physics and astrophysics, 2002, no. 1, p.49.

[11] H. Kleinert, Gauge theory of dislocation meting, Phys. Lett. 89A (6).

[12] P. Radjaraman, Soliton and instanton in quantum theories of a field, M. The world: 1985;

[13] P. Godard, J. Nuyts, D. Olive, Gauge theory and magnetic charge, Nuclear Physics B125 (1977) 1-28.

[14] Z. P. Serr, Lie Group and Lie Algebra, M. Mir, 1969;

Received January 16, 2007

\section{ĐỐI NGẪU TRONG QUÁ TRÌNH BIẾN DẠNG KHÔNG GIAO HOÁN VÀ BẢN CHẤT TOPO CỦA TÍCH PHÂN CHEREPANOV-RICE}

Trong bài này chúng tôi chỉ ra rằng trong quá trình biến dạng không giao hoán sẽ tồn tại đồng thời hai loại biến dạng: biến dạng đặt tăi và biến dạng cất tải. Biến dạng thực là tổ hợp của hai loại biến dạng trên. Tiêu chuẩn phá huỷ J phản ánh đặc trưng to po của môi trường, tức là nó phản ánh đặc trưng đối xứng của môi trường trong quá trình phá huỷ. Có thể nói rằng trong quá trình phá huỷ năng lượng được giải phóng ra không liên tục mà gián đoạn. Tình hình này được phản ánh qua số to po Q hoặc chỉ số cất tải. 